\title{
Les enjeux de la formation continue des enseignants dans les Centres Régionaux des Métiers de l'Education et de la Formation (CRMEF) au Maroc
}

\section{Mili Abdesselam (1) - Erouihane Jaouad (2) - Toubi Lahcen (3)}

(1) Professeur Agrégé, Docteur en sciences de l'éducation, Directeur du CRMEF GC

(2) Professeur Assistant, Docteur en Histoire, Directeur du CRMEF DA

(3) Professeur Assistant, Docteur en sciences de l'éducation, Formateur au CRMEF GC

\section{Résumé}

La formation continue des enseignants au Maroc a pris des formes multiples et diversifiées sur le plan organisationnel et fonctionnel. Aussi le recours à la formation continue reste-t-il, malgré tout, faible et n'atteint généralement pas les résultats escomptés. Actuellement, les $\mathrm{CRMEF}^{1}$ sont les seules entités chargées de la formation du personnel du $\mathrm{MEN}^{2}$ (enseignants, administrateurs, techniciens...). Cette mission, parmi d'autres, attribuée aux CRMEF est dans sa phase d'implémentation. En effet, la formation continue des enseignants, qui constitue la mission phare des CRMEF, n'a pas encore pris l'élan qu'il faut. S'agit-il d'un blocage institutionnel, financier, ou pédagogique ? Rappelons que, selon le décret en vigueur, les CRMEF et les AREF ${ }^{3}$ doivent coordonner entre eux pour réaliser cette mission. Or, il est plus facile de dire que de faire. Nous allons montrer à travers l'expérimentation du dispositif de formation continue de la langue française durant l'année scolaire 2014/2015, l'importance de la prise en compte de certaines dispositions notamment : la qualité du dispositif de formation, le type de motivation attendu par les bénéficiaires, le processus de changement des représentations des concernés, pour garantir une formation qui pourrait avoir un impact positif sur le système. La nature de la nouvelle formule de formation est permanente, hebdomadaire et assurée au CRMEF selon un créneau horaire négocié.

Mots clés : la formation continue- CRMEF- carrière professionnelle- ingénierie de la formation- impact- dispositif de formation

\footnotetext{
${ }^{1}$ Les centres régionaux des métiers de l'Education et de la Formation.

${ }^{2}$ Ministère de l'Education Nationale et de la Formation professionnelle.

${ }^{3}$ Académie Régionale de l'Education et la Formation.
} 


\title{
The challenges of continuing education for teachers in the Regional Centers for Education and Training Skills (CRMEF) in Morocco
}

\begin{abstract}
:
The teacher in-service training in Morocco has experienced multiple and diverse forms at the organisational and functional levels. The resort to continuous training remains, nevertheless, weak and does not generally achieve the desired results. Currently, $\mathrm{CRMEF}^{4}$ are the only entities responsible for the training of National Education Ministry $\left(\mathrm{MEN}^{5}\right)$ staff (teachers, administrators, technicians ...). This mission, among others, attributed to CRMEF is in its implementation phase. Indeed, the training of teachers, which constitutes the core mission, has not yet taken momentum. Is it an institutional or pedagogical deadlock? Let's not forget that, according to the current decree, that $\mathrm{CRMEF}$ and $\mathrm{AREF}^{6}$ must coordinate with each other to achieve this mission. Yet, easier said than done. Through experimentation of the training device of the French language in the academic year 2014/2015, we will show the importance of taking into consideration certain provisions in particular: the quality of the training system, the type of motivation expected by the beneficiaries, the process of the change of representations of the people concerned, to ensure a training that could have a positive impact on the system. The nature of the new training formula is permanent, weekly and ensured at the CRMEF on a negotiated time table.
\end{abstract}

Keywords: continuing education - CRMEF - professional career - training engineering - impact - training system

\footnotetext{
${ }^{4}$ Regional centres for education and teaching skills

${ }^{5}$ Ministry of National Education and vocational training

${ }^{6}$ Regional Academy for Education and Training
} 


\section{1- Introduction}

Les ressources humaines sont amenées à s'adapter et à évoluer dans leur contexte professionnel ; elles sont confrontées à l'évolution de l'environnement social, économique, technologique etc. La veille et la vigilance constitue l'un des paramètres fondamentaux du management actuel des ressources humaines. C'est ainsi que les institutions investissent énormément pour l'actualisation des savoirs et des savoir-faire de leurs employés. C'est l'une des conditions à réaliser pour que ces institutions soient en concordance avec les objectifs escomptés. La formation continue est l'un des moyens permettant d'assurer aux ressources humaines l'adaptation et l'action sur l'environnement. Les études montrent bien cette relation dans le monde de l'entreprise (Guyot, Mainguet, \& Van Hae, 2003), (OECD, 2000), (Burnel, 2010), mais aussi dans le monde de l'éducation (Carlier, Renard, \& Paquay, 2000) (RIFEFF, 2008). La formation continue des enseignants, quant à elle, vise l'amélioration des apprentissages et la réussite des apprenants, par la transformation des pratiques professionnelles des enseignants. Cette transformation se manifeste par un ensemble de changements attendus dans les pratiques d'enseignement et les pratiques enseignantes. Bien que la formation continue soit toujours préconisée par les enseignants, beaucoup d'entre eux sont insatisfaits de son apport. Pourquoi ne répond -t-elle pas à leurs besoins professionnels ou personnels ? S'agit-il d'un problème organisationnel, institutionnel ou motivationnel ? De plus, la formation cible une minorité des d'enseignants et est limitée dans le temps, à travers des programmes spécifiques, le plus souvent, financés par les organismes internationaux notamment les bailleurs de fond. La formule adoptée par les départements du MEN au niveau central ou au niveau régional, quant à elle, reste malgré les efforts déployés, éparpillée et non finalisée. En fait, l'organisation de la formation est réduite à la passation d'un contenu préparé à l'avance. Généralement, les facteurs déterminants ne sont pas pris en considération. Bien qu'une ingénierie de formation soit bien faite, il est possible que la formation parte en fumée suite à un problème organisationnel.

C'est dans ce sens qu'une question nous interpelle: comment peut-on assurer une formation continue de qualité, répondant aux besoins des concernés et à la demande de l'institution pour impacter les apprentissages ? C'est à travers l'étude des rapports réalisés dans le cadre de la formation continue et les bilans de l'expérimentation du dispositif de formation de la langue française dans certaines AREF en coordination avec les CRMEF, durant l'année scolaire 2014/2015, que nous allons mettre au point, en les dégageant, les principes et les facteurs clés qui puissent garantir une formation continue afin de maximiser, en leur donnant un sens, les objectifs escomptés dans le système éducatif marocain. Pour approcher cette problématique, nous 
nous proposons de définir les enjeux de la formation et son importance, ensuite nous allons décrire le cadre scientifique sur lequel s'appuie cet article. Enfin, nous procéderons à l'analyse des différents rapports concernant la conduite des formations continues et l'expérimentation du dispositif de formation pour en tirer les résultats qui peuvent servir à une planification juste de la formation continue.

\section{2- Place et contexte de la formation continue des enseignants au Maroc}

Depuis les années 60, la formation continue, au Maroc, a pris plusieurs formes. Elle est organisée, institutionnalisée dans des établissements de formation $\left(\mathrm{ENS}^{7}, \mathrm{CPR}^{8}, \mathrm{CFI}^{9}, \mathrm{COPE}^{10}\right)$ pour que les lauréats bénéficient d'un statut particulier après avoir réussi toutes les évaluations. C'est le cas du $2^{\text {ème }}$ cycle de l'ENS, du cycle spécial dans les CPR, du cycle de formation des inspecteurs, du cycle de formation-des chargés de l'orientation ou de la planification, du cycle de l'agrégation. Ces cycles, bien qu'ils aboutissent aux objectifs escomptés, ciblent une minorité des d'enseignants et exigent un investissement sur le plan humain et financier. Certaines formules de ces cycles ont été abandonnées ou en cours de disparition. D'autres formes de formation continue, limitées dans le temps (1 à 4 jours), programmées selon des projets ponctuels, spécifiques, et ciblant quelques régions et quelques enseignants, restent le plus souvent au stade de l'expérimentation. Ces types de formation sont organisés soit par des organismes extérieurs (nationaux, internationaux) et les bailleurs de fond ou par les départements ministériels (centraux ou régionaux). Les expériences tentées, dans ce sens, soit pour la formation soit pour le recyclage (ou la remise à niveau) des enseignants, prennent fin avec la fin du projet. De plus, les interventions sont souvent éparses et ponctuelles. Les besoins sont quelquefois déterminés en fonction des objectifs des organismes et non en fonction des besoins réels. Nous trouverons aussi certaines initiatives prises par des groupements syndicaux ou associatifs pour répondre à un besoin immédiat et ponctuel (préparation aux examens de la promotion interne par exemple). De plus, la formation est réalisée par des formateurs qui focalisent la formation sur un thème ponctuel qui se trouve parfois déplacé par rapport aux objectifs recherchés.

\footnotetext{
${ }^{7}$ Ecole Normale Supérieure

${ }^{8}$ Centre Pédagogique Régional

${ }^{9}$ Centre de Formation des Inspecteurs

${ }^{10}$ Centre d'Orientation et de Planification de l'Education
} 
Rappelons aussi que l'encadrement des enseignants est confié aux inspecteurs ${ }^{11}$ dans le cadre de leur mission d'accompagner, d'encadrer et de superviser les activités pédagogiques par l'organisation de journées pédagogiques, de séminaires entre enseignants. Cependant, faute de temps et de disponibilité pour certains et de compétences pour d'autres, cette mission prend de plus en plus un caractère administratif pour l'attribution de la note de promotion.

Parmi les expériences nationales, celles avancées durant la période 1999- 2002, ciblant les enseignants du second cycle. Bien qu'une analyse de besoin ait été réalisée dans certaines disciplines, les modules à la carte ont été préparés en didactique, psychologie, pédagogie, audiovisuel, discipline de spécialité et informatique en lien avec l'enseignement de certaines disciplines (Mawfik, 2005). Ces modules n'ont pas été reconduits pour les raisons suivantes :

- Absence d'un cadre institutionnel

- Ambiguïté dans les rôles des inspecteurs et formateurs/ administration de l'ENS, administration des académies

- Disponibilité du matériel

- Disponibilité des enseignants à la formation (Mawfik, 2005).

Dans le cadre de la généralisation des technologies de l'Information et de la Communication en éducation, le programme GENIE a permis, durant les années 2006-2013, à lancer la formation des cadres pédagogiques. Le programme vise la formation des enseignants pour une utilisation effective des outils TIC mis à leur disposition. La stratégie a préconisé l'approche de la formation en cascade pour aboutir à cette formation de masse (Abouhanifa, Drissi , Kabba, \& Talbi , 2009). Bien que des bilans aient été réalisés pour améliorer le dispositif de formation, «l'implication des enseignants dans le processus d'intégration des TICE et leur manque de motivation reflètent clairement leur résistance au changement. L'une des justifications avancées face à cette attitude, est qu'il n'existe pas de motivation extrinsèque qui pourrait garantir leur engagement. Ils évoquent notamment la prise en compte de leur formation et du développement professionnel dans l'évolution de leurs carrières. Le soutien et la reconnaissance institutionnels semblent également faire défaut » (Messaoudi \& Talbi, 2012).

\footnotetext{
${ }^{11}$ Les articles 4, 8, 10 du décret 2.02.254 du 10 février 2003 relatif au statut de personnel du MEN, l'article $\mathrm{N}^{\circ} 6$ du décret Royal du 02 Février 1967 précisent la mission des inspecteurs
} 
La vision de la formation continue dans le programme d'urgence ${ }^{12}$ est plus globale ; elle vise à travers le projet E3. P1 de mettre en place le nouveau système de formation continue au niveau de l'enseignement scolaire (MENESFCRS, 2009-2012, p. 32). Le schéma directeur stratégique repose sur les éléments suivants :

- Mettre en place des structures de gestion de la formation continue au niveau régional ;

- Faire un bilan de compétence des personnels pour évaluer l'écart entre les compétences actuelles et les compétences telles qu'elles figurent dans le REC (référentiel des emplois et compétences),

- Développer d'autres modes de formation continue : formation à distance, formation à la carte, formation par la pratique, formation tutorée, formation de proximité (concept de caravane de formation) ateliers de partage, autoformation,

- Disposer de textes juridiques et d'outils permettant de lier la formation continue à la promotion et à la gestion de carrière (modalités de certifier les formations, modalités de les rendre obligatoires, les outils de motivation ...),

- Disposer d'un système d'information opérationnel pour la gestion et le pilotage de la FC,

- Intégrer la formation continue au sein d'un système global pour la gestion des fonctions et des compétences : recrutement, redéploiement, mouvement, réforme des systèmes, la promotion et la gestion de la carrière

- Élaborer des modules de FC (expérimentation et validation)

- Définir des normes (durée, période, fréquence) de formations continues

- Définir des programmes de formation continue du personnel, en coordination avec les inspecteurs, notamment dans le cadre des zones pédagogiques

- Réaliser de 1125000 jours de formation continue en 2009, 1375000 en 2010, 1625000 en 2011 et 1875000 en 2012.

Les formations ont ciblé les thèmes suivants ${ }^{13}: 1^{\prime}$ Approche par les compétences, les innovations pédagogiques, la langue amazighe, le manuel scolaire, l'enseignement préscolaire, l'enseignement

\footnotetext{
${ }^{12}$ Le plan d'urgence se définit comme cadre de référence et vise à donner « un nouveau souffle » à la Charte Nationale de l'Education et de la Formation.

${ }^{13}$ Le caractère national notamment l'approche par cómpéténces, les nouveaux programmes et manuels scolaires, le programme GENIE, les nouveaux dispositifs d'évaluation et des examens, l'enseignement de la langue Amazighe...En 2006 a été adoptée l'approche dite «effet démultiplicateur» qui consiste à former, au niveau central, des experts chargés
} 
technique, commercial et industriel, l'enseignement de l'EPS. La formation en pédagogie de l'intégration a pris le devant sur tous les projets de formation mis en place sur le plan organisationnel, effectif des enseignants formés...

A travers ce qui a été avancé ci-dessus, l'analyse de certains rapports présentés au MEN montre :

- L'existence de dispersion et de diversité de la formation, ce qui rend obscure voire confuse l'image du pilotage et de la gestion des projets de la formation continue ;

- L'absence d'un plan national de formation continue. La majorité des formations sont ponctuelles et non généralisables. Ce qui a pour une conséquence l'accentuation de l'inégalité et l'iniquité des apprentissages entre les apprenants. Certains enseignants n'ont bénéficié d'aucune formation depuis leur sortie du centre de formation initiale ;

- L'absence d'une entité instituée, spécialisée et structurée pour organiser et abriter la formation continue. Ce qui a pour conséquence, une gestion de la formation momentanée basée le plus souvent sur une analyse et une exploitation des besoins non professionnels et sur une proposition non pertinente des contenus par rapport aux vrais besoins. De plus, l'information ne cible que certains enseignants. À ceci s'ajoute l'apparition des problèmes et des réclamations ayant une relation avec les conditions d'accueil : infrastructures de formation, restauration, hébergement, indemnisation des formateurs, motivation des bénéficiaires...

- La formation n'est pas intégrée dans le processus de gestion des institutions scolaires : le volume horaire annuel alloué à la formation continue des enseignants, les périodes prévues pour la formation, le remplacement des enseignants, l'implication occasionnelle des directeurs des écoles, le respect du temps et le rythme des apprentissages des apprenants.

- La disponibilité des intervenants et des formateurs pour assurer l'harmonisation de l'encadrement des axes de formation. A ceci, s'ajoute le problème de la spécialisation des formateurs assurant certaines formations continues. Il est rare qu'on sollicite à cet effet des personnes ressources extérieures. Ce qui, par conséquent, renforce le désengagement et la démotivation des bénéficiaires de la formation.

L'affaire de la formation continue est avant tout une volonté politique. Hausser le niveau nécessite un engagement et un investissement à long terme. Elle doit être intégrée comme un pilier 
fondamental dans cette optique de réforme. La formation continue du personnel enseignant «n'a pas toujours eu la place qu'elle mérite dans les plans de développement de l'éducation, comme en témoigne la part insignifiante qui lui est réservée dans les budgets annuels. ». (50 de développement humain; Comité directeur, 2006, p. 34). Pourtant, le levier 13 de la Charte Nationale de l'Éducation et de la Formation recommande des sessions de requalification plus approfondies, intervenant au moins tous les trois ans et des sessions de courte durée de 30 heures de formation, programmée annuellement pour l'amélioration des compétences et l'élévation du niveau des pratiques d'enseignement. Le CSE, dans son rapport de 2008 précise de « Consacrer des sessions de formation continue aux méthodes, outils et techniques de l'évaluation pédagogique, au profit des enseignants, des inspecteurs et des directeurs d'établissements, de manière à assurer leur adhésion effective dans le processus de suivi et de mesure des acquis scolaires et d'évaluation du rendement de l'établissement scolaire dans lequel ils travaillent ». (CSE, 2009, p. 30). Enfin, le rapport CSEFRS 2014 montre le rôle important de la formation continue en précisant qu'elle «se doit de permettre aux enseignants de compléter et de perfectionner leur formation pour qu'ils soient capables de suivre et de s'adapter aux réformes que connaît le système éducatif. »(CSEFRS; (INESEFRS), 2014, p. $34)$.

\section{3- Repères théoriques de la formation continue}

\section{- L'enseignant : un adulte en situation de formation}

La formation continue des enseignants, bien qu'elle prenne des formes diversifiées (la formation permanente, le recyclage, le perfectionnement professionnel, ou encore le développement professionnel (Perrenoud, 1996)), elle s'adresse à l'adulte comme un «être en situation » (MASCIOTRA, 2004), car l'enseignant revit la situation professionnelle autrement. Elle est abordée « comme une forme de travail humain en situation, comme « une action située ». (Carlier, Renard, \& Paquay, 2000, p. 146). Son engagement dans la formation est conditionné-par sa perception de l'apport personnel et professionnel de ladite formation. C'est une formation en cours de l'exercice qui vise « soit la compensation d'un déficit de la formation initiale, soit l'adaptation au changement, soit le perfectionnement, soit la préparation à la reconversion» (Karsenti, 2007, p. 126). Aujourd'hui, la formation continue ou permanente prend une dénomination dans le processus de l'éducation tout au long de la vie « life long learning » (VANISCOTTE, 2000, pp. 184-185). Or, toute formation qui n'aiderait pas les enseignants à résoudre certains problèmes vécus sur le terrain 
ne peut être acceptée et constitue l'objet de déclenchement de la résistance au changement. Beaucoup, d'entre eux réclament l'adaptation des contenus à la réalité de l'école et fuient les discours théoriques que le formateur envisage comme moyen de véhiculer le savoir. (Mili \& Chiadli, 2013). Car «la formation à la pédagogie des adultes ayant ses propres mécanismes, règles, dispositifs, elle ne peut être pratiquée sans la formation préalable de ceux qui s'impliqueront dans la formation continue. » (Karsenti, 2007, p. 69).

\section{- Les approches usuelles de la formation continue des enseignants}

L'inspiration des théories des apprentissages pour expliquer le processus enseignementapprentissage est utilisée aussi dans la conduite des formations. Le cognitivisme, constructivisme, le socioconstructivisme et l'interactionnisme sont souvent pris comme référence pour expliquer l'adulte en situation de formation. Beaucoup de modèles de formation font référence à l'interactionnisme et au socioconstructivisme pour proposer les démarches organisationnelles et pédagogiques pour assurer la formation. Ces modèles sont complétés par d'autres ayant référence aux sciences humaines comme le modèle MARC (motifs, attentes, rôles, contenus) qui offre un cadre particulièrement intéressant, parce qu'il souligne les principales questions que soulèvent les adultes apprenants.

\section{- L'importance de la formation continue}

L'importance de la formation continue réside dans le fait qu'elle permet à l'enseignant d'améliorer ses compétences professionnelles et de pouvoir agir d'une manière efficace lors de la conduite des apprentissages. L'objectif est donc que chaque enfant puisse avoir un enseignant qualifié, ce qui lui assure une réussite scolaire et une équité à l'éducation pour tous. Le besoin de recycler après une formation initiale est incontournable pour évoluer dans la carrière et être acteur de la réussite scolaire. Car, la formation initiale, quelle que soit sa qualité, n'est jamais définitive face à l'évolution des connaissances et des techniques (Paré,1983). Elle doit être programmée tout au long de la carrière professionnelle (CSEFRS, 2015, p. 34) de l'enseignant. Son impact sur le système est un autre point d'intérêt qui se manifeste dans la qualité de l'enseignement et les meilleures acquisitions des connaissances des élèves. Ceci permet au système éducatif non seulement de concurrencer les autres systèmes, mais participer au développement de la nation. Le recyclage des enseignants a aussi son importance lorsqu'on introduit une réforme ou une innovation. Ce qui nécessite un changement possible de leurs pratiques auxquelles ils n'étaient pas habitués. 


\section{4- Le contexte de la formation dans les CRMEF}

L'attribution de la mission de la formation continue aux CRMEF est impulsée par la volonté politique d'instituer, de rationaliser et de généraliser la formation tenant compte des bilans et des lacunes constatées dans la gestion de la formation continue. Il s'agit d'une rupture avec le système de la formation classique. L'article 33 du décret $N^{\circ}$ 2-11-672 du 23 décembre 2011 portant création et organisation des CRMEF montre le rôle primordial de ces centres dans la formation continue du personnel du MEN. Il précise que « Les $\mathrm{CRMEF}^{14}$ participent à l'exécution et la mise en œuvre de la stratégie du secteur de l'enseignement dans le domaine de la formation continue, à travers la participation dans la mise en place des plans d'action annuel. Ces centres veillent sur l'organisation et l'encadrement des sessions de la formation continue et le complément d'expertise au profit des cadres de la communauté de l'enseignement, mais aussi les autres communautés du personnel du secteur de l'enseignement scolaire, à ceux-ci s'ajoutent les cadres exerçant dans l'enseignement préscolaire, l'enseignement scolaire privé. En plus ces centres participent dans l'élaboration des modules de formation, le suivi et l'évaluation des formations programmées à l'échelle des AREF concernées suite à leurs demandes ».

Les raisons du choix des CRMEF pour l'organisation de la formation continue sont :

- La formation dans les CRMEF est instituée par un cadre juridique ;

- Les CRMEF permettent d'assurer le lien recherché entre la formation initiale et la formation continue. Ils disposent d'un dispositif de formation à caractère académique et professionnel ;

- Les CRMEF disposent de l'infrastructure et de la logistique permettant l'organisation de la formation continue (bibliothèque, plate-forme, salle spécialisée, matériel didactique...);

- Les CRMEF ont pour vocation la recherche pédagogique scientifique (Centres de recherche, laboratoire...);

- C'est un lieu des experts : internes ou externes. Les CRMEF sont ouverts à toutes les compétences et aux personnes ressources.

L'expérimentation du dispositif de formation continue des professeurs (primaire-collège) dans les CRMEF est lancée durant l'année scolaire 2014/2015. Ce projet s'inscrit dans le cadre PREF .... La formation se compose de dix modules à raison d'une trentaine d'heures par module.

\footnotetext{
${ }^{14}$ Centres régionaux des métiers d'éducation et de formation (CRMEF)
} 
Chaque CRMEF et AREF ont organisé la formation en fonction des conditions dont ils disposent. La formation modulaire est étalée sur trois mois, à raison de 2 à 3 séances de 3 heures par mois. Ces séances sont programmées au CRMEF avec les enseignants ayant des après-midis ou des matinées réservées aux rencontres avec l'inspecteur. La formation est assurée par des équipes constituées chacune d'un formateur et d'un inspecteur pour éviter les dissonances qui peuvent apparaître volontairement ou involontairement entre le formateur et l'évaluateur. Cette expérimentation permet de tester la mise en place d'un système de coordination entre le CRMEF et l'AREF et les modalités d'implication de leur personnel administratif et pédagogique (respect mutuel et de responsabilité, engagement, relation de partenariat, confiance, négociation, etc.). Selon le rapport présenté par les organisateurs au ministère, la formation a rencontré des difficultés :

- «Soutien timide aux coordonnateurs et implication discontinue pour la réalisation du projet

- Coordination avec les AREF est timide, non acquise

- Rompre avec les modalités classiques (restauration, indemnités, concentration de la formation en 2 ou 3 jours, refus de certains acteurs dans certains AREF de participer à la concrétisation de l'expérimentation. » (MEN, 2015)

- Le manque de préparation et de communication et vis-à-vis du projet, $50 \%$ des participants se sont engagés.

- Certains CRMEF ont organisés la formation tardivement dû à un problème de coordination entre ces centres et les AREF.

- L'esprit d'acquisition des indemnités relatives à la formation règne lors de la programmation des formations avec les intervenants.

- Peu d'enseignants motivés par la formation.

\section{5- Les facteurs clés d'une formation continue des enseignants}

Les études et les rapports traitant de la problématique de la formation continue s'accordent à souligner son importance, à s'interroger sur les conditions de sa programmation et sur l'impact auquel elle aboutit. Les éléments qui donnent un statut à la formation continue sont le contexte juridique, l'ingénieure pédagogique, la carrière professionnelle et l'impact sur le terrain.

\section{1- Cadre juridique de la formation continue des enseignants (institutionnalisation) :}

Les formations assurées sont souvent sous forme de projets non institués. Elles ne sont pas obligatoires. L'intéressé, par la formation, n'aura pas de contrepartie à part un certificat délivré dans 
les meilleurs des cas par l'entité organisatrice. Il n'y a aucune réglementation, ni consigne qui oblige son suivi et son impact sur le terrain. Ce manquement juridique n'assure en aucun cas la pérennité de la majorité des formations. Certaines formations continues ne sont qu'une liquidation d'un budget accumulé surtout si elle est programmée à la fin de l'année budgétaire. De plus, ce manquement, constitue un estompage au niveau de la mission des planificateurs et des intervenants.

Actuellement, les CRMEF disposent de cadre juridique (voir article 33 du décret $N^{\circ}$ 2-11-672 du 23 décembre 2011 ci-dessus), mais il est dans sa phase embryonnaire. Le CSEFRS rappelle cette tendance en précisant que « La formation continue et la qualification tout au long de la vie professionnelle offre aux acteurs éducatifs une occasion renouvelée de perfectionnement professionnel, de mise à niveau et de suivi de l'évolution de leur domaine. A ce propos, il faudra, à court terme, préparer et mettre en œuvre, en coordination avec les académies, les centres de formation et les universités, des stratégies de formation continue spécifique aux diverses catégories de personnels, dotées de plans de formation et répondant à leurs besoins effectifs ». (CSEFRS, 2015, p. 34)

\section{2- Ingénierie pédagogique}

Une formation de qualité repose sur un véritable dispositif de formation répondant à des besoins clairs et pertinents par rapport aux objectifs de la formation, qu'il s'agisse de développement de compétences professionnelles ou l'introduction d'une innovation ou d'une réforme. La politique est définie par le MEN selon un calendrier annuel. Les CRMEF et les AREF ensemble devront œuvrer à déterminer cette politique à partir des observations et des constats réalisés sur le terrain ; car l'analyse des besoins en absence des concernés est une perte de temps et d'énergie. C'est ainsi que Labesse $(2008$, p.5) précise que « les analyses des besoins de formation assurent la pertinence des activités de formation pour les populations ciblées qui sont constituées par les professionnels ou les types de professionnels concernés par la nécessité d'accroître la qualité et l'efficience du travail ». Le recours à l'analyse des besoins est d'une importance capitale, car il permet d'identifier et de cibler les causes du dysfonctionnement apparent ou caché d'un système, l'anticipation des actions face à un environnement en pleine mutation ou le renforcement de compétences professionnelles des enseignants. A cet égard, «il conviendra de prospecter la possibilité de bénéficier de l'expertise et du savoir-faire professionnel, pédagogique et de gestion des personnels de l'éducation, de la formation et de la recherche à la retraite ; ceux-ci apporteront leur appui à la formation continue et aux efforts de professionnalisation des différents acteurs éducatifs et administratifs. Cela implique 
de prévoir des incitations pour ceux qui contribueront à cette mission. ». (CSEFRS, 2015, p. 34). En fait, le véritable dispositif de formation repose sur :

- L'identification de la source des besoins (autrement chez qui) : est-ce que chez l'apprenant, ou chez l'enseignant ou chez l'institution. Le travail quotidien de la classe permet d'apercevoir ce besoin.

- L'analyse des besoins par des experts et des professionnels qui utilisent des outils et des moyens pour détecter ces besoins avec les concernés.

- L'élaboration d'un plan national de la formation et des programmes régionaux contextualisés.

- Une coordination entre le CRMEF, l'AREF et les centres de recherche pour assurer une formation de qualité répondant à des besoins ciblés et étudiés.

- Une programmation tenant compte du temps scolaire, de la proximité (formateurs mobiles), l'homogénéité des équipes...

\section{3- Gestion de la carrière professionnelle :}

Il s'agit des droits et devoirs des bénéficiaires de la formation. En fait, le caractère de la non obligation et de l'absence de motivation encouragent les enseignants à fuir la formation. Pour renforcer l'engagement et l'acceptabilité, il est nécessaire de lier la formation continue aux motivations des concernés. En général, la formation continue « ne donne aucun avantage de carrière ou de statut, ce qui peut avoir pour conséquence l'absence de motivation et d'engagement des enseignants. La formation continue est pourtant partout un droit». (Carlier, Renard, \& Paquay, 2000, p. 188). Nous remarquons souvent que les enseignants contractuels exerçant dans le secteur de l'enseignement privé sont demandeurs de la formation continue contrairement aux enseignants titularisés dans le secteur public. En fait, la demande à la formation est morale car c'est un besoin professionnel. Certains pays comme l'Espagne, le Portugal et l'Islande soumettent l'avancement dans la carrière à la participation aux actions de la formation continue. (Carlier, Renard, \& Paquay, 2000, p. 188). Une autre forme de motivation consiste à faire le lien entre la formation continue et la passation au grade supérieur (passeport professionnel au canada, certificat d'aptitude présentée tous les 5 ans aux USA). Car, «tout changement dépend de l'aspect motivationnel (intention de faire), de l'aspect professionnel (la compétence pour le faire) et l'aspect opérationnel (les conditions pour faire). En effet, un enseignant satisfait et motivé pour une pratique a tendance à s'impliquer, à 
s'investir car l'innovation pour lui est une réponse à un besoin. Or, un enseignant motivé ne suffit pas, car il doit avoir les compétences nécessaires pour assurer le bon déroulement des pratiques enseignantes. » (Mili \& Chiali, 2014). Quelle que soit sa forme, la motivation à la formation est d'une importance capitale dans la stratégie politique de la formation des enseignants. Comme le précise ici le CSEFRS «La politique de formation continue des enseignants demeure insuffisante à plusieurs égards. Sur le plan législatif et contrairement aux préconisations de la Charte, la formation continue n'est prise en compte ni dans la promotion ni dans le mouvement du personnel enseignant, d'où le désintérêt et la démotivation chez les enseignants pour des formations sans incidence notable sur l'amélioration de leur carrière professionnelle ». (CSEFRS; (INESEFRS), 2014, p. 35). L'étude menée par Evrard, C. Dubar cité par (Malglaive, 1990, p. 261) s'inscrit dans cette lignée. Il note que « le désir de formation semble influencé par deux facteurs importants : d'une part, le fait d'entrevoir des possibilités d'amélioration socio-professionnelle, et, d'autre part, une attitude positive vis-à-vis de l'école dans la mesure où celle-ci est jugée susceptible d'influencer cette promotion ».

\section{4- Impact de la formation continue :}

L'impact de la formation sur le changement des pratiques d'enseignement dépend du degré de la transférabilité des savoirs appropriés en formation en savoir d'action sur le terrain. Si la formation ne prend pas en considération le contexte de l'exercice de l'enseignant, il y a de forte chance que le contenu dispensé au cours de la formation, aussi riche soit-il, soit abandonné. Une formation, aussi, sans suivi de son impact sur le terrain ne garantit pas la pérennité du changement souhaité ; car, les pratiques acquises en formation nécessitent une adaptation quand elles sont mises en œuvre sur le terrain. Donc, il est nécessaire de consolider cette formation par : les visites en classe, l'assistance des personnes ressources, la mise en place des activités de formation de proximité, et l'accompagnement des enseignants. En fait, « les enseignants apparaissent volontiers «traditionnalistes » et souvent méfiants envers les tentatives de transformation de leur métier. Ils ne rejettent pas automatiquement les efforts pour «améliorer» leurs pratiques et les adapter aux dernières innovations pédagogiques; cependant, s'ils changent, c'est toujours en intégrant le nouveau à l'ancien et en incorporant l'innovation aux traditions établies ». M. Tardif et C. Lessard (2004) cité par (Guibert \& Troger, 2012). Le renforcement de la formation par l'exploitation de nouvelles technologies de l'information et de la communication est toujours une voie prometteuse. La mise en place des plates-formes en e-learning est l'une des formes de la formation à distance qui, malgré les efforts, reste en deçà des objectifs souhaités. 


\section{5- Organisation de la formation :}

- La rationalisation : la bonne gouvernance de la formation est sa rationalisation. Car la formation continue exige des dépenses difficilement contrôlables.

- Le temps de la formation : c'est le temps hors apprentissage des apprenants. Il est peutêtre programmé dans les demi-journées de rencontres pédagogiques des enseignants. Le CRMEF et les annexes peuvent accueillir ces enseignants suivant un programme établi en coordination avec l'AREF. Les formateurs du CRMEF et les inspecteurs assureront la formation et l'encadrement selon un dispositif préparé pour les séances de formation. Ainsi, chaque enseignant aura l'occasion d'assister à des cours (théorique ou pratique) dans le CRMEF.

- La mobilité des formateurs : pour les enseignants exerçant dans des établissements ruraux, et qui n'ont pas la possibilité de se déplacer pour assister aux séances de la formation continue dans les CRMEF. Un système de déplacement des formateurs vers les établissements pour assurer la formation en compagnie avec les inspecteurs et les enseignants ressources.

- La gestion des effectifs : le grand problème que rencontrent les concepteurs et les organisateurs de la formation est l'effectif important des enseignants à former. Selon les chiffres avancés par le CSEFRS, le nombre d'enseignants est de l'ordre de 225802 dans l'enseignement public (126446 au primaire, 55964 au collège, 43392 au qualifiant) et 63712 dans les écoles privées (CSEFRS; (INESEFRS), 2014, p. 30). Les CRMEF et les AREF sont amenés à assurer la formation à chaque enseignant exerçant dans les établissements de la région. Quels critères peut-on utiliser pour cibler les enseignants à former? Une question complexe et compliquée dans la mesure où le profil de ces enseignants est hétérogène. Le ciblage est l'une des méthodes qui peuvent être réalisées en confrontant l'analyse des besoins et les objectifs attendus de la formation. D'autres alternatives peuvent être proposées telle que la mise en relation de la validation des acquis selon la formation modulaire et la promotion professionnelle du bénéficiaire de la formation. Le ciblage des enseignants accompagnateurs ${ }^{15}$ peut être une opportunité aussi pour les former afin d'assurer le suivi et l'accompagnement des enseignants sur le terrain.

- La mise en place d'un observatoire ou un learnscape ${ }^{16}$ de l'évolution des métiers de l'éducation et de la formation dont le but de créer un climat d'échange et de recherche entre les différents acteurs du système éducatif.

\footnotetext{
${ }^{15}$ Note ministérielle $\mathrm{N}^{\circ} 15 / 134$ du 11 Décembre 2015.

16 «Un learnscape est écosystème dans lequel les salariés se rencontrent, partagent des idées, se tiennent au courant, racontent des histoires, collaborent, s'entraident, résolvent des problèmes, conceptualisent et formalisent des bonnes pratiques, apprennent, enseignent, servent leurs clients, nouent des partenariats et se rassemblent en communauté d'intérêts » (Parmentier, 2014, p. 338) 


\section{Conclusion}

Bien que le ministère ait pour souci d'instaurer un système de formation continue des enseignants pour la réussite scolaire, et en dépit de toutes les actions programmées, la formation continue semble souffrir des insuffisances aux niveaux de l'attachement et de la représentation de l'entité qui est responsable de la formation. Si la formation est instituée dans les CRMEF, la volonté politique et la stratégie de la mise en œuvre est dans sa phase embryonnaire. Aujourd'hui, les enseignants ont besoin d'être reformés et les élèves ont besoin d'enseignants compétents ou de qualité. Les CRMEF disposent actuellement d'un atout pour relancer la formation continue. Ils seront considérés comme des lieux spécialisés pour la formation et le développement des ressources humaines qu'elles soient novices ou expertes. Chaque cadre trouve dans ces entités l'occasion d'exprimer son potentiel. C'est aussi l'endroit pour développer l'andragogie et tout le processus qui en découle. Les cursus de formation seront homogénéisés et harmonisés pour garantir aux élèves un apprentissage équitable. C'est le défi du système éducatif. Vu les transformations que va rencontrer le cursus scolaire des apprenants au niveau du type de Bac et la langue d'enseignement choisie, les besoins de la formation, actuellement, sont accrues et urgents. Quant aux CRMEF, ils sont dans l'obligation de faire évoluer la conception de la formation par « le passage d'un système fermé (on forme pour former) à un système ouvert à son environnement (on forme pour répondre aux besoins de l'organisation et de ses membres, on forme pour investir). (GERARD, LAVENDHOMME, \& ROEGIERS, 1997, p. 237). Les formateurs doivent intégrer dans leurs conceptions l'apport de l'expertise extérieure dans la formation dans le but de répondre à différents besoins d'amélioration de la qualité de la formation et de la pratique enseignante, de la recherche, de la relève du corps enseignant et du système éducatif. 


\section{Références}

50 ans de développement humain; Comité directeur. (2006). Le Maroc possible: Une offre de débat pour une ambution collective. CD rom: Systèmes Educatifs, Savoir, Technologies et Innovation. Casablanca: Sapress.

Abouhanifa , S., Drissi , A., Kabba, M., \& Talbi , M. (2009, Septembre). Programme GENIE au Maroc : TICE et développement professionnel. (16), p. 1. Récupéré sur mathéTICE: http://revue.sesamath.net/spip.php?article233

Burnel, P. (2010). La formation professionnelle continue: Obligations financières: Volume 2. Malmaison: Lamy.

Carlier, G., Renard, J.-P., \& Paquay, L. (2000). La formation continue des enseignants. Bruxelles: De Boeck.

Carlier, G., Renard, J.-P., \& Paquay, L. (2000). La formation continue des enseignants: Enjeux, innovation et réflexivité. Bruxelles: De boock.

CSE. (2009). Rapport synthétique: Programme National d'Evaluation des Aquis PNEA 2008.

CSEFRS. (2015). Vision stratégique de la réforme 2015-2030: Pour une école de l'équité, de la qualité et de la promotion. Rabat: CSEFRS.

CSEFRS; (INESEFRS). (2014). ATLAS GRAPHIQUE ET CARTOGRAPHIQUE DE LA DÉCENNIE DE LA CHARTE NATIONALE DE L'EDUCATION ET DE LA FORMATION.

CSEFRS; (INESEFRS). (2014). Rapport analytique: La mise en œuvre de la Charte Nationale d'Education et de Formation 2000-2013: Acquis, déficits et défis. Rabat: CSEFRS.

GERARD, F.-M., LAVENDHOMME, E., \& ROEGIERS, X. (1997). La formation continue : pour quoi ? Dans F.-M. GERARD, E. LAVENDHOMME, \& X. ROEGIERS, Analyser une action d'éducation ou de formation. Bruxelles: De Boeck Université.

Guibert, P., \& Troger, V. (2012). Peut-on former des enseignants. Paris: Armand Colin.

Guyot, J. L., Mainguet, C., \& Van Hae, B. (2003). La formation professionnelle continue: l'individu au coeur des dispositifs. Bruxelles: De Boeck et Larcier s.a.

Karsenti, T. (2007). La formation continue dans la francophonie:: Canada: AUF. 
Malglaive, G. (1990). Enseigner à des adultes. Paris: PUF.

MASCIOTRA, D. (2004). Être, penser et agir en situation d'adversité : perspective d'une théorie du connaître ou de l'énaction. Dans P. J. (dir.), Constructivisme - Choix contemporains. Hommage à Ernst von Glasersfeld, Sainte-Foy. Quebec: Presses universiitaires.

Mawfik, N. (2005). Récupéré sur https://www.google.com/?gws_rd=ssl\#q=ENS+Rabat+Mawfik+\%282\%29

MEN, s. d. (2015). Projet d'appui à la formation des formateurs de français. 2015: MEN.

MENESFCRS. (2009-2012). Récupéré sur

http://planipolis.iiep.unesco.org/upload/Morocco/Morocco_Programme_Urgence_portefeu ille.pdf

Messaoudi, F., \& Talbi, M. (2012, Mars 2012). Réussir l'intégration des TICE au Maroc : regard sur le déploiement de la stratégie nationale GENIE. (A. EPI, Éd.) Récupéré sur La revue électronique de l'EPI: http://www.epi.asso.fr/revue/articles/a1203e.htm

Mili, A., \& Chiadli, A. (2013). La formation des enseignants et transformation des pratiques d'enseignements. Sciencelib.

Mili, A., \& Chiali, A. (2014). Modèle d'analyse des pratiques enseignantes lors de l'instauration d'une réforme curriculaire. Science.Lib.

Norcross, \& Norcross, J. C. (2012). La relation thérapeutique. Dans B. L. Duncan, S. D. Miller, B. E. Wampold, \& M. A. Hubble, L'essence du changement. Bruxelles: De boeck.

OECD. (2000). De la formation initiale à la vie active Faciliter les transitions. Paris: OECD.

Parmentier, C. (2014). Pour tout réussir le métier de formateur. Paris: Eyrolles.

RIFEFF. (2008). Former les enseignants du XXIème siècle dans toute la francophonie. Université Clermont2: AUF.

VANISCOTTE, F. (2000). Fonctions et dispositifs de la formation continue des enseignants: tendances européennes. Dans G. Carlier, J.-P. Renard, \& L. PAQUAY (EDS), La formation continue des enseignants: Enjeux, innovation et réflexivité. Bruxelles: De Boeck Université. 